Manuscript

\title{
Thromboembolien und Thrombophilie in der Schwangerschaft
}

[Thromboembolism and thrombophilia in pregnancy]

Michael Nagler ${ }^{1,2}$; Anne Angelillo-Scherrer ${ }^{1,2}$

\author{
Affiliations: \\ ${ }^{1}$ University Clinic of Hematology and Central Hematology Laboratory, Inselspital, Bern \\ University Hospital, University of Bern, CH-3010 Bern, Switzerland \\ ${ }^{2}$ Department of Clinical Research, University of Bern, CH-3010 Bern, Switzerland
}

$\begin{array}{ll}\text { Correspondence to: } & \text { michael.nagler@insel.ch } \\ \text { Text word count: } & 1981 \\ \text { Tables: } & 5\end{array}$




\section{Zusammenfassung}

Venöse Thromboembolien, meist in Form von Bein-/Beckenvenenthrombosen und Lungenembolien auftretend, stellen immer noch eine der führenden Ursachen mütterlicher Morbidität und Mortalität in der Schwangerschaft dar. Eine effektive Prophylaxe und sichere Therapie sind daher wichtige Massnahmen, um Komplikationen zu vermeiden und jungen Frauen eine gefahrlose Schwangerschaft zu ermöglichen. Im folgenden Artikel beschreiben wir Prinzipien der Risikoabschätzung sowie die Diagnose und Therapie venöser Thromboembolien. Zudem diskutieren wir das peripartale Management der Antikoagulation, die Risikoabschätzung im Hinblick auf östrogenhaltige Kontrazeptiva, sowie den Einfluss thrombophiler Risikofaktoren auf Schwangerschaftskomplikationen.

\section{Summary}

[Thromboembolism and thrombophilia in pregnancy]

Venous thromboembolism is still one of the main factors contributing to pregnancy-related morbidity and mortality in developed countries. Effective prophylaxis and safe treatment of venous thromboembolism are therefore important measures to reduce maternal complications. In the present article, we report on risk factors for venous thromboembolism and discuss principles of an effective risk assessment. We present current recommendations for diagnosis of venous thrombosis and pulmonary embolism, review prophylaxis and treatment with low molecular weight heparins, and discuss management of peripartum anticoagulation. In addition, we consider aspects of thrombophilia and pregnancy complications, recurrent miscarriage in particular. Furthermore, we discuss the risk assessment with regard to oestrogen-containing contraceptives. 


\section{Einleitung}

Die ersten Beschreibungen von Venenthrombosen stammen aus dem 18. Jahrhundert. Es handelte sich um schwangere Patienten und man nahm einen „Milchstau“ als Ursache an ${ }^{1}$. Die Schwangerschaft ist daher der älteste bekannte Risikofaktor für venöse Thromboembolien und deren Folgen waren gefürchtet. Unter venösen Thromboembolien werden nicht nur tiefe Beinvenenthrombosen der unteren Extremität sowie Lungenembolien zusammengefasst, sondern auch seltenere Lokalisationen wie Hirnvenen- (Sinusvenen-) Thrombosen, nicht jedoch oberflächliche Thrombophlebitiden. Zu Beginn des zwanzigsten Jahrhunderts traten in etwa 8 von 1000 Geburten Thrombosen oder Lungenembolien auf, welche in einem Drittel der Fälle tödlich endeten ${ }^{1}$. Verbesserungen in Prophylaxe und Therapie reduzierten das Auftreten venöser Thromboembolien auf etwa 1 Ereignis in 1000 Geburten (ca. 1\% fatal). Obwohl die absoluten Zahlen gering sind stellen venöse Thromboembolien immer noch eine der führenden Ursachen mütterlicher Morbidität und Mortalität in den Industrienationen dar ${ }^{2}$. Eine effektive Prophylaxe und sichere Therapie venöser Thromboembolien sind daher wichtige Massnahmen, um Komplikationen zu vermeiden und jungen Frauen eine gefahrlose Schwangerschaft zu ermöglichen.

Im folgenden Artikel diskutieren wir Risikofaktoren und Prinzipien der Risikoabschätzung und geben Empfehlungen für prophylaktische Massnahmen. Zudem beschreiben wir die Diagnose und Behandlung venöser Thromboembolien. Weiterhin diskutieren wir den Einfluss von thrombophilen Risikofaktoren auf Thromboembolien und Schwangerschaftskomplikationen.

\section{Thrombosen und Lungenembolien in der Schwangerschaft}

Im Vergleich zu Nicht-Schwangeren tragen Schwangere ein etwa fünffach erhöhtes Risiko für venöse Thromboembolien, erleiden öfter Beckenvenen-Thrombosen, Lungenembolien und ein post-thrombotisches Syndrom ${ }^{3}$. Bekannt ist zudem, dass die Thrombosen aufgrund der anatomischen Gegebenheiten sehr oft links auftreten. Das Risiko ist nochmals höher in der Postpartal-Periode, besonders in den ersten drei Wochen. Da jedoch nur ein kleiner Teil der Schwangeren Thromboembolien erleidet, ist für eine effektive Prophylaxe eine gute Risikoabschätzung notwendig. In Tabelle 1 sind die wichtigsten Risikofaktoren mit ihrem Einfluss dargestellt. Auffällig ist, dass klinische Faktoren einen hohen Stellenwert besitzen. Der weitaus wichtigste Risikofaktor sind frühere Thromboembolien, hierfür gibt es auch die besten epidemiologischen Daten. Je nach Untersuchung wird eine bis zu 25-fache Risikoerhöhung angenommen ${ }^{1}$. Auch bei adipösen Frauen, älteren Schwangeren und bei Immobilisation ist das Risiko deutlich erhöht. Es gilt zu beachten, dass bestimmte Risikofaktoren nur für die 
Postpartal-Periode wichtig sind (z.B. das Auftreten eine postpartalen Blutung). Demgegenüber gibt es im Fall der labormässigen, thrombophilen Risikofaktoren praktisch nur Fallkontrollstudien mit den damit verbundenen Limitationen. Es wird angenommen, dass nur bei sogenannten „Hochrisiko-Thrombophilien“ eine relevante Risikoerhöhung allein durch den Laborwert besteht. Hierunter werden vor allem die homozygoten oder kombinierten Störungen gezählt und die ausgeprägten Mängel von Protein S, Protein C und Antithrombin. Eine Ausnahme stellt das erworbene Antiphospholipid-Antikörpersyndrom dar. Bei diesem besteht eine deutliche Erhöhung des thromboembolischen Risikos.

\section{Risikoabschätzung und Monitoring im Hinblick auf venöse Thromboembolien}

Die Risikoabschätzung hat zum Ziel, diejenigen Schwangeren zu identifizieren, welche von einer medikamentösen Prophylaxe profitieren. Aufgrund der oben genannten methodischen Limitationen und der Dominanz bestimmter klinischer Risikofaktoren wird die Bestimmung der Thrombophilieparameter nur in Ausnahmefällen zur Risikoabschätzung herangezogen. Alle Empfehlungen wissenschaftlicher Fachgesellschaften rücken die klinischen Faktoren in den Vordergrund, insbesondere das Vorhandensein früherer venöser Thromboembolien ${ }^{4-8}$. Eine gute, aber bereits etwas ältere Anleitung bieten die Empfehlungen des Royal College of Obstetricians and Gynaecologists ${ }^{4}$, welche alle klinischen Risikofaktoren in einer Checkliste auflisten (eine adaptierte Version findet sich in Tabelle 2). Eine Bestimmung von Thrombophilieparametern wird nur bei bekannter Hochrisikothrombophilie in der Familie empfohlen (Homozygote Faktor V-Leiden oder Prothrombingenmutation, kombinierte Defekte, ausgeprägte Protein S-, Protein C- oder Antithrombinmangel, allenfalls Dysfibrinogenämie) sowie bei Hinweisen auf ein Antiphospholipid-Antikörper Syndrom. Wir empfehlen in der klinischen Praxis ein Vorgehen entsprechend Tabelle 3. Eine medikamentöse Prophylaxe sollten Schwangere mit früheren venösen Thromboembolien erhalten, mit HochrisikoThrombophilien oder mit mehreren klinischen Risikofaktoren.

Eine Ausnahme stellt das Antiphospholipid-Antikörper Syndrom dar. Dieses stellt eine Hochrisiko-Situation dar, nicht nur für venöse und arterielle Thromboembolien, als auch für bestimmte Schwangerschaftskomplikationen. Bei klinischem Verdacht sollte hier eine entsprechende Labordiagnostik veranlasst werden. Ein Verdacht sollte bei Symptomen eines systemischen Lupus erythematodes geäussert werden (Allgemeinsymptome, Hautveränderungen, Blutbild), beim Auftreten sowohl arterieller als auch venöser Thromboembolien als auch bei bestimmten Schwangerschaftskomplikationen. Zur Diagnose eines Antiphospholipid-Antikörper Syndroms müssen nicht nur bestimmte Laborkriterien erfüllt 
sein ${ }^{9}$, sondern auch klinische Kriterien: stattgehabte venöse oder arterielle Thromboembolien oder bestimmte Schwangerschaftskomplikationen. Diese sind definiert als: (1) mindestens ein unerklärtes Absterben eines Fetus in oder jenseits der 10. Gestationswoche, mit normaler fetaler Morphologie (Ultraschall oder direkte Untersuchung des Fetus) oder (2) eine oder mehrere frühzeitige Geburten eines morphologisch normalen Neugeborenen vor der 34 . Schwangerschaftswoche wegen (a) Eklampsie oder schwerer Präeklampsie, oder (b) nachgewiesener Plazentainsuffizienz, bzw. (3) drei oder mehrere konsekutive Spontanaborte ohne erkennbare Ursache vor der 10. Gestationswoche. Schwangere mit AntiphospholipidAntikörper Syndrom sollten nicht nur eine prophylaktische bis therapeutische Antikoagulation mit niedermolekularen Heparinen erhalten, sondern auch eine Behandlung mit Aspirin $100 \mathrm{mg}$ (von der 10. bis 36. Schwangerschaftswoche).

Ein weiterer Sonderfall ist das Hyperstimulationssyndrom. Hier sollte eine prophylaktische Antikoagulation bis zur 13. Schwangerschaftswoche durchgeführt werden, beim schweren Hyperstimulationssyndrom sogar bis 3 Monate nach Sistieren der Symptome.

\section{Diagnose venöser Thromboembolien in der Schwangerschaft}

Die diagnostische Aufarbeitung von Schwangeren mit postulierten venösen Thromboembolien ist durch verschiedene Punkte erschwert: eine Reihe von klinischen Zeichen sind bei Schwangeren physiologisch vorhanden (z.B. Schwellungen und Beschwerden in den Beinen sowie Tachypnoe bei Anstrengung), die D-Dimere steigen im Verlauf der Schwangerschaft an, eine Exposition durch computertomographische Strahlung soll vermieden werden, und Beckenvenenthrombosen sind häufig und können bei einer Duplexsonographie der Beine verpasst werden. Demgegenüber ist eine korrekte Diagnose unbedingt angezeigt, um eine übermässige Behandlung mit den verbundenen Risiken für Mutter und Kind zu vermeiden. Die Duplexsonographie der Beinvenen ist darum der Grundpfeiler der Diagnostik, sowohl im Hinblick auf Bein- und Beckenvenenthrombosen als auch der Lungenembolie ${ }^{10}$. Klinische Entscheidungshilfen und die Bestimmung der D-Dimere spielen nur eine untergeordnete Rolle. Falls der klinische Verdacht auf eine Beckenvenenthrombose oder eine Lungenembolie besteht und in der Duplexsonographie eine Thrombose der Beinvenen dargestellt wird, kann die Diagnose ohne weitere Massnahmen als bestätigt gelten. Falls jedoch keine Thrombose gezeigt wird, müssen weitere diagnostische Massnahmen eingeleitet werden. Im Hinblick auf eine Lungenembolie kommt soweit verfügbar eine Ventilations-/Perfusionsszintigraphie in Frage. Im Hinblick auf eine Beckenvenenthrombose wird entsprechend Verfügbarkeit eine Venographie oder ein Angio-CT empfohlen. 


\section{Therapie venöser Thromboembolien in der Schwangerschaft}

In den meisten Ländern sind zwei Medikamente für die Behandlung venöser Thromboembolien in der Schwangerschaft zugelassen: unfraktioniertes Heparin (z.B. Liquemin $®$ ) und bestimmte niedermolekulare Heparine (z.B. Dalteparin - Fragmin $\left.{ }^{\circledR}\right)$. Sie treten nicht in die Muttermilch über und haben in Tierstudien keine Missbildungen gezeigt. Vitamin K-Antagonisten sind aufgrund der teratogenen Wirkung streng kontraindiziert. Für neue, direkte orale Antikoagulantien wie Rivaroxaban (Xarelto ${ }^{\circledR}$ ), Apixaban (Eliquis $®$ ), Dabigatran (Pradaxa $®$ ) oder Edoxaban (Lixiana ${ }^{\circledR}$ ) existieren keinerlei klinische Daten. Aufgrund der niedrigeren Blutungsrisiken und der subkutanen Applikation wird man sich in den meisten Fällen für niedermolekulare Heparine entscheiden. Insbesondere im Fall von Dalteparin und auch Enoxaparin gibt es im Hinblick auf Wirksamkeit und Sicherheit bei Schwangeren inzwischen gute klinische Daten. Jedoch sollte jede Indikation gut geprüft werden, da eine extrem seltene Komplikation, die Heparin-induzierte Thrombozytopenie, potentiell lebensbedrohliche Konsequenzen haben kann. Basierend auf aktuellen Empfehlungen haben wir in Tabelle 4 Dosisempfehlungen zusammengefasst ${ }^{4,5,8,11}$. In der Prophylaxe kommt in der Regel eine prophylaktische Dosis zur Anwendung und nur bei hohem Risiko (Antiphospholipid-Syndrom, dauherhafte Antikoagulation) eine intermediäre oder therapeutische Dosis. Im Hinblick auf die Therapie venöser Thromboembolien wird - aufgrund der besseren Datenlage - häufig zwar eine zweimal tägliche Gabe empfohlen. Für viele Schwangere ist dies jedoch nicht möglich, sodass auch die einmal tägliche Gabe eine gute Alternative ist. In der Behandlung venöser Thromboembolien kann nach einem Monat auf eine etwas reduzierte Dosis (ca. 75\%) gewechselt werden, z.B. bei befürchteten Blutungskomplikationen. In diesem Fall empfehlen wir Dalteparin 150 E/kg Körpergewicht oder Enoxaparin $1.5 \mathrm{mg} / \mathrm{kg}$ Körpergewicht ${ }^{12}$. Die prophylaktische Behandlung sollte bis 6 Wochen postpartal weitergeführt werden, die therapeutische Behandlung für 3 bis 6 Monate; mindestens aber bis 6 Wochen postpartal. Einige Experten empfehlen bei therapeutischer Antikoagulation die monatliche Bestimmung der anti Xa Werte (siehe Tabelle 4). Bei Unverträglichkeiten steht als Reservemedikament Fondaparinux (Arixtra ${ }^{\circledR}$ ) zur Verfügung ${ }^{13}$.

Eine Behandlung mit Aspirin 100 mg kommt nur zur Prophylaxe einer Präeklampsie in Frage sowie bei vorliegendem Antiphospholipid-Antikörpersyndrom in Kombination mit niedermolekularen Heparinen.

Wie soll die Antikoagulation im Umfeld der Geburt gesteuert werden? Wir empfehlen, beim Einsetzen der ersten Wehen auf die Applikation niedermolekularer Heparine zu verzichten. 
Diese sollten jedoch - sobald von Seiten der Blutungssituation vertretbar - rasch wieder eingesetzt werden. Bei besonders hohem thromboembolischen Risiko (mechanische Herzklappe, venöse Thromboembolie in den vergangenen 4 Wochen) empfehlen wir eine Überbrückung mit unfraktioniertem (intravenösem) Heparin. Vor einer Peridualanästhesie sollte ein Abstand von 12 Stunden (prophylaktische bis intermediäre Dosis) bzw. 24 Stunden (therapeutische Dosis) eingehalten werden.

\section{Risikoabschätzung im Hinblick auf östrogenhaltige Kontrazeptiva}

Im klinischen Alltag stellt sich die häufig die Frage, ob östrogenhaltige Kontrazeptive aufgrund einer Risikosituation weiter eingenommen werden dürfen. Aufgrund der sehr seltenen Ereignisse stehen keine Interventionsstudien und nur eingeschränkt Beobachtungsstudien zur Beantwortung dieser Fragen zur Verfügung. Wichtig ist, es die Unsicherheiten mit dem Patienten zu diskutieren. In jedem Fall gilt, dass östrogenhaltige Kontrazeptiva unter fortgeführter Antikoagulation nicht nur eingenommen werden dürfen, aufgrund der (zum Teil vorliegenden) teratogenen Wirkung der Antikoagulantien sogar eingenommen werden müssen. Tabelle 5 gibt hier eine Hilfestellung zur Entscheidungsfindung. Rein gestagenhaltige Präparate (auch transdermal applizierte) erhöhen das thromboembolische Risiko nicht. Dagegen haben transdermal applizierte kombinierte Präparate (Pflaster, Vaginalring) sogar ein höheres Risiko als peroral eingenommene Kombinationspräparate ${ }^{14}$. Bei Kombinationspräparaten ist weiterhin zu beachten, dass Präparate der dritten Generation mit einem höheren Risiko verbunden sind als Präparate der zweiten Generation.

\section{Thrombophilien und Schwangerschaftskomplikationen}

Aborte und Totgeburten sind ausgesprochen belastende Ereignisse für Paare mit Kinderwunsch, insbesondere bei wiederholtem Auftreten. Aufgrund pathologischer Untersuchungen, theoretischer Überlegungen, und infolge klinischer Fall-Kontroll-Studien werden Störungen der Hämostase und Mikrozirkulation als wichtige Risikofaktoren angesehen. Zudem ist die antithrombotische Behandlung in speziellen Situationen (AntiphospholipidAntikörper Syndrom) und bei anderen Schwangerschaftskomplikationen (HELLP-Syndrom, Präeklampsie) eine etablierte Therapie. Ein vermuteter Zusammenhang zwischen dem Vorhandensein von Thrombophilien und Schwangerschaftskomplikationen konnte jedoch in später durchgeführten, grossen prospektiven Kohortenstudien nicht bestätigt werden ${ }^{15,16}$. Es wurden grosse Interventionsstudien durchgeführt, welche bei Patientinnen mit wiederholten Aborten den Effekt von niedermolekularen Heparinen und/oder Aspirin untersucht haben. Auch diese konnten die Wirksamkeit dieser Massnahmen nicht bestätigen ${ }^{17}$. Etwas weniger klar ist 
die Datenlage im Hinblick auf Patienten mit bekannten Thrombophilien und rezidivierenden Aborten. Eine randomisierte Untersuchung beobachtete weniger Aborte unter der Gabe niedermolekulare Heparine gegenüber Aspirin (jedoch ohne Placebo-Gruppe) ${ }^{18}$. Demgegenüber konnte kein Effekt niedermolekularer Heparine gegenüber Placebo in einer weiteren, randomisierte Studien gezeigt werden ${ }^{19}$. Einschränkend muss hinzugefügt werden, dass es sich in den meisten Fällen um „Niedrigrisiko-Thrombophilien“ gehandelt hat. Es muss zudem beachtet werden, dass sowohl unter niedermolekularen Heparinen, als auch unter Aspirin klar ein erhöhtes Blutungsrisiko ${ }^{20}$. Zusammenfassend unterstützen die vorhandenen Daten aktuell weder die Behandlung rezidivierender Aborte mit niedermolekularen Heparinen noch mit Aspirin. Unter Kenntnis der Risiken und der fehlenden Daten kann sich nach guter Diskussion mit der Patientin im Einzelfall (z.B. Hochrisiko-Thrombophilie) jedoch auch dafür entschieden werden - insbesondere im Hinblick auf das damit verbundene thromboembolische Risiko.

Ein Spezialfall ist das Antiphospholipid-Syndrom. Dieses ist klar mit bestimmten Schwangerschaftskomplikationen verbunden (siehe oben). Obwohl auch in diesem Fall aufgrund der Seltenheit und ethischer Schwierigkeiten keine randomisierten Interventionsstudien existieren, wird eine Behandlung sowohl mit niedermolekularen Heparinen als auch mit Aspirin 100 mg von allen Fachgesellschaften empfohlen (siehe Tabelle 3). Es wird angenommen, dass damit das Risiko sowohl für venöse und arteriell Thromboembolien, als auch für Schwangerschaftskomplikationen vermindert werden kann.

Weitere Spezialfälle sind das HELLP-Syndrom und die Präeklampsie. In beiden Fällen ist die Behandlung mit Aspirin 100 mg zur Prophylaxe und Therapie etabliert und durch gute klinische Daten gesichert.

\section{Zusammenfassung}

Schwangere tragen ein etwa fünffach erhöhtes Risiko für venöse Thromboembolien. Diese sind mit einer erheblichen Morbidität und Mortalität verbunden und gefährden sowohl die Mutter als auch das Kind. Für eine effektive Prophylaxe ist eine gute Risikoabschätzung notwendig. Hier stehen klinische Risikofaktoren im Vordergrund, insbesondere früher stattgehabte Ereignisse. Die Diagnose von Venenthrombosen und Lungenembolien ist gegenüber Nicht-Schwangeren erschwert und die Duplexsonographie ist das Instrument der ersten Wahl, auch im Hinblick auf Lungenembolien und Beckenvenenthrombosen. Präparate der ersten Wahl sind niedermolekulare Heparine, sowohl in der Prophylaxe als auch der Therapie. Mit der Ausnahme des Antiphospholipid-Antkörper Syndroms, der Präeklampsie und dem HELLP-Syndrom haben 
Antikoagulanzien und Antiaggregantien in der Therapie rezidivierender Aborte derzeit keinen

Stellenwert.

\section{Referenzen}

1. Guimicheva B, Czuprynska J, Arya R. The prevention of pregnancy-related venous thromboembolism. British journal of haematology 2015;168:163-74.

2. Kourlaba G, Relakis J, Kontodimas S, Holm MV, Maniadakis N. A systematic review and meta-analysis of the epidemiology and burden of venous thromboembolism among pregnant women. International journal of gynaecology and obstetrics: the official organ of the International Federation of Gynaecology and Obstetrics 2016;132:4-10.

3. Greer IA. CLINICAL PRACTICE. Pregnancy Complicated by Venous Thrombosis. The New England journal of medicine 2015;373:540-7.

4. Gynaecologists RCoOa. Reducing the Risk of Thrombosis and Embolism During Pregnancy and the Puerperium. Green-top Guideline 2009.

5. Bates SM, Greer IA, Middeldorp S, et al. VTE, thrombophilia, antithrombotic therapy, and pregnancy: Antithrombotic Therapy and Prevention of Thrombosis, 9th ed: American College of Chest Physicians Evidence-Based Clinical Practice Guidelines. Chest 2012;141:e691S-736S.

6. American College of O, Gynecologists Women's Health Care P. ACOG Practice Bulletin No. 138: Inherited thrombophilias in pregnancy. Obstetrics and gynecology 2013;122:706-17.

7. McLintock C, Brighton T, Chunilal S, et al. Recommendations for the diagnosis and treatment of deep venous thrombosis and pulmonary embolism in pregnancy and the postpartum period. Aust N Z J Obstet Gynaecol 2012;52:14-22.

8. European Society of G, Association for European Paediatric C, German Society for Gender M, et al. ESC Guidelines on the management of cardiovascular diseases during pregnancy: the Task Force on the Management of Cardiovascular Diseases during Pregnancy of the European Society of Cardiology (ESC). European heart journal 2011;32:3147-97.

9. Miyakis S, Lockshin MD, Atsumi T, et al. International consensus statement on an update of the classification criteria for definite antiphospholipid syndrome (APS). Journal of thrombosis and haemostasis : JTH 2006;4:295-306.

10. Barco S, Nijkeuter M, Middeldorp S. Pregnancy and venous thromboembolism. Seminars in thrombosis and hemostasis 2013;39:549-58.

11. Yarrington CD, Valente AM, Economy KE. Cardiovascular Management in Pregnancy: Antithrombotic Agents and Antiplatelet Agents. Circulation 2015;132:1354-64.

12. Bates SM, Middeldorp S, Rodger M, James AH, Greer I. Guidance for the treatment and prevention of obstetric-associated venous thromboembolism. J Thromb Thrombolysis 2016;41:92-128.

13. Nagler M, Haslauer M, Wuillemin WA. Fondaparinux - data on efficacy and safety in special situations. Thrombosis research 2012;129:407-17.

14. Lidegaard O, Nielsen LH, Skovlund CW, Lokkegaard E. Venous thrombosis in users of non-oral hormonal contraception: follow-up study, Denmark 2001-10. BMJ 2012;344:e2990. 
15. Rodger MA, Walker MC, Smith GN, et al. Is thrombophilia associated with placentamediated pregnancy complications? A prospective cohort study. Journal of thrombosis and haemostasis : JTH 2014;12:469-78.

16. Coppens M, Folkeringa N, Teune MJ, et al. Outcome of the subsequent pregnancy after a first loss in women with the factor V Leiden or prothrombin 20210A mutations. Journal of thrombosis and haemostasis : JTH 2007;5:1444-8.

17. Kaandorp SP, Goddijn M, van der Post JA, et al. Aspirin plus heparin or aspirin alone in women with recurrent miscarriage. The New England journal of medicine 2010;362:1586-96.

18. Gris JC, Mercier E, Quere I, et al. Low-molecular-weight heparin versus low-dose aspirin in women with one fetal loss and a constitutional thrombophilic disorder. Blood 2004;103:36959.

19. Rodger MA, Hague WM, Kingdom J, et al. Antepartum dalteparin versus no antepartum dalteparin for the prevention of pregnancy complications in pregnant women with thrombophilia (TIPPS): a multinational open-label randomised trial. Lancet 2014;384:1673-83.

20. Middeldorp S. New studies of low-molecular-weight heparin in pregnancy. Thrombosis research 2015;135 Suppl 1:S26-9.

21. Jacobsen AF, Skjeldestad FE, Sandset PM. Ante- and postnatal risk factors of venous thrombosis: a hospital-based case-control study. Journal of thrombosis and haemostasis : JTH 2008;6:905-12.

22. Bramham K, Retter A, Robinson SE, Mitchell M, Moore GW, Hunt BJ. How I treat heterozygous hereditary antithrombin deficiency in pregnancy. Thromb Haemost 2013;110:5509.

23. Nagler M, Surbek D, Angelillo-Scherrer A. Thrombophilie in Schwangerschaft und Wochenbett. Gynäkologie 2015:6-13. 
Tabelle 1: Risikofaktoren venöser Thromboembolien in der Schwangerschaft

\begin{tabular}{|c|c|c|}
\hline \multirow[t]{2}{*}{ Faktor } & \multicolumn{2}{|c|}{ Ungefähre Risikoerhöhung* } \\
\hline & Präpartal & Postpartal \\
\hline \multicolumn{3}{|l|}{ Klinische Risikofaktoren } \\
\hline Frühere Venenthrombosen/ Lungenembolie $\left(\mathrm{OR}^{\#}\right)$ & \multicolumn{2}{|c|}{5 bis 20} \\
\hline Adipositas (BMI > $\left.30 \mathrm{~kg} / \mathrm{m}^{2} ; \mathrm{OR}^{\#}\right)$ & 10 & 3 \\
\hline Alter über 35 Jahren $\left(\mathrm{OR}^{\#}\right)$ & 1.5 & 2 \\
\hline \multicolumn{3}{|l|}{ Schwangerschafts-assoziierte Risikofaktoren } \\
\hline Mehrlingsschwangerschaften $\left(\mathrm{OR}^{\#}\right)$ & \multicolumn{2}{|c|}{3} \\
\hline Elektive Sectio caesare $\left(\mathrm{OR}^{\#}\right)$ & - & 1.3 \\
\hline Notfall Sectio caesare $\left(\mathrm{OR}^{\#}\right)$ & - & 2 bis 4 \\
\hline Immobilisation $\left(\mathrm{OR}^{\#}\right)$ & 8 & 11 \\
\hline Assistierte Befruchtung $\left(\mathrm{OR}^{\#}\right)$ & 4 & 2 \\
\hline Präeklampsie $\left(\mathrm{OR}^{\#}\right)$ & 1 & 3 bis 6 \\
\hline Postpartale Blutung $\left(\mathrm{OR}^{\#}\right)$ & - & 4 \\
\hline \multicolumn{3}{|l|}{ Angeborene Thrombophilien } \\
\hline Heterozygote Faktor V-Leiden Mutation & \multicolumn{2}{|c|}{ Geringe Risikoerhöhung } \\
\hline Heterozygote Prothrombingen-Mutation & \multicolumn{2}{|c|}{ Geringe Risikoerhöhung } \\
\hline Homozygote Faktor V-Leiden Mutation & \multicolumn{2}{|c|}{ Relevante Risikoerhöhung } \\
\hline Homozygote Prothrombingen-Mutation & \multicolumn{2}{|c|}{ Relevante Risikoerhöhung } \\
\hline Compound heterzygote Prothrombingen-/Faktor V-Leiden Mutation & \multicolumn{2}{|c|}{ Relevante Risikoerhöhung } \\
\hline Protein C-Mangel $(<50 \%)$ & \multicolumn{2}{|c|}{ Geringe Risikoerhöhung } \\
\hline Protein S-Mangel $(<50 \%)$ & \multicolumn{2}{|c|}{ Geringe Risikoerhöhung } \\
\hline Dysfibrinogenämie & \multicolumn{2}{|c|}{$?$} \\
\hline \multicolumn{3}{|l|}{ Erworbene Thrombophilien } \\
\hline Antiphospholipid-Antikörper Syndrom (OR $)$ & \multicolumn{2}{|c|}{5 bis 16} \\
\hline
\end{tabular}


Tabelle 2: Empfehlungen des Royal College of Obstetricians and Gynaecologists zur

Risikoabschätzung im Hinblick auf venöse Thromboembolien in der Schwangerschaft (adaptiert nach $\left.^{4}\right)^{*}$

\begin{tabular}{ll}
\hline Risikofaktor & Punkte \\
\hline Frühere, idiopathische venöse Thromboembolien & $\mathbf{3}$ \\
Frühere venöse Thromboembolien unter Östrogenen & $\mathbf{3}$ \\
Frühere, provozierte venöse Thromboembolien & 2 \\
Adipositas (BMI > 35 kg/m2) & 2 \\
Begleiterkrankungen & 2 \\
Section caesare & 2 \\
Chirurgische Eingriffe <6 Wochen & 2 \\
Immobilisation & 2 \\
Hochrisiko-Thrombophilie & 2 \\
Bekannte Thrombophilie & 1 \\
Positive Familienanamnese & 1 \\
Alter > 35 Jahre & 1 \\
Rauchen & 1 \\
Ausgeprägte Varikosis & 1 \\
Prä-Eklampsie & 1 \\
Dehydratation, Hyperemesis & 1 \\
Mehrlingsschwangerschaften & 1 \\
Lange Geburt & 1 \\
Postpartale Blutung & 1 \\
Infektion & 1 \\
\hline$*$ Ab & 1 \\
\hline
\end{tabular}

* Ab drei Punkten wird eine medikamentöse Prophylaxe empfohlen 
Tabelle 3: Hausinterne Empfehlungen zur medikamentösen Prophylaxe venöser

Thromboembolien in der Schwangerschaft (adaptiert nach ${ }^{4-6,8,11}$ )

\begin{tabular}{|c|c|c|}
\hline Kontext & Präpartal* & Postpartal\# \\
\hline \multicolumn{3}{|l|}{ Frühere Thromboembolien } \\
\hline Nicht provoziert (idiopathisch) & $\begin{array}{l}\mathrm{NMH}+\text { in prophylaktischer } \\
\text { Dosierung }\end{array}$ & $\begin{array}{l}\mathrm{NMH}+\text { in prophylaktischer } \\
\text { Dosierung }\end{array}$ \\
\hline $\begin{array}{l}\text { Unter Östrogenen/ in } \\
\text { Schwangerschaft }\end{array}$ & $\begin{array}{l}\mathrm{NMH}+\text { in prophylaktischer } \\
\text { Dosierung }\end{array}$ & $\begin{array}{l}\mathrm{NMH}+\text { in prophylaktischer } \\
\text { Dosierung }\end{array}$ \\
\hline Provoziert (nach Operationen) & Klinisches Monitoring & $\begin{array}{l}\mathrm{NMH}+\text { in prophylaktischer } \\
\text { Dosierung }\end{array}$ \\
\hline Multiple Ereignisse & $\begin{array}{l}\mathrm{NMH+} \text { in prophylaktischer, } \\
\text { intermediärer oder } \\
\text { therapeutischer Dosis } \Uparrow\end{array}$ & $\begin{array}{l}\mathrm{NMH+} \text { in prophylaktischer, } \\
\text { intermediärer oder } \\
\text { therapeutischer Dosis } \uparrow\end{array}$ \\
\hline \multicolumn{3}{|l|}{ Niedrigrisiko-Thrombophilien ${ }^{\circ}$} \\
\hline Keine Thromboembolien & Klinisches Monitoring & Klinisches Monitoring \\
\hline Positive Familienanamnese\$ & Klinisches Monitoring & $\begin{array}{l}\mathrm{NMH}+\text { in prophylaktischer } \\
\text { Dosierung }\end{array}$ \\
\hline Stattgehabte Thromboembolien & $\begin{array}{l}\mathrm{NMH+} \text { in prophylaktischer, } \\
\text { intermediärer oder } \\
\text { therapeutischer Dosis } \uparrow\end{array}$ & $\begin{array}{l}\mathrm{NMH+} \text { in prophylaktischer, } \\
\text { intermediärer oder } \\
\text { therapeutischer Dosis } \uparrow\end{array}$ \\
\hline \multicolumn{3}{|l|}{ Hochrisiko-Thrombophilien\& } \\
\hline Keine Thromboembolien & $\begin{array}{l}\text { Klinisches Monitoring oder } \\
\text { prophylaktische Antikoagulation }\end{array}$ & $\begin{array}{l}\mathrm{NMH+} \text { in prophylaktischer } \\
\text { Dosierung }\end{array}$ \\
\hline Positive Familienanamnese $\$$ & $\begin{array}{l}\mathrm{NMH}+\text { in prophylaktischer } \\
\text { Dosierung }\end{array}$ & $\begin{array}{l}\mathrm{NMH+} \text { in prophylaktischer } \\
\text { Dosierung }\end{array}$ \\
\hline Stattgehabte Thromboembolien & $\begin{array}{l}\mathrm{NMH+} \text { in prophylaktischer, } \\
\text { intermediärer oder } \\
\text { therapeutischer Dosis } \uparrow\end{array}$ & $\begin{array}{l}\mathrm{NMH+} \text { in prophylaktischer, } \\
\text { intermediärer oder } \\
\text { therapeutischer Dosis } \uparrow\end{array}$ \\
\hline Antiphospholipid-Antikörper Syndrom & $\begin{array}{l}\mathrm{NMH}+\text { in prophylaktischer oder } \\
\text { intermediärer Dosis und Aspirin } \\
100 \mathrm{mg}(10 . \text { bis } 36 . \mathrm{SSW})\end{array}$ & $\begin{array}{l}\mathrm{NMH}+\text { in prophylaktischer oder } \\
\text { intermediärer Dosis }\end{array}$ \\
\hline Klinische Risikofaktoren** & $\begin{array}{l}\mathrm{NMH}+\text { in prophylaktischer } \\
\text { Dosierung }\end{array}$ & $\begin{array}{l}\mathrm{NMH}+\text { in prophylaktischer } \\
\text { Dosierung }\end{array}$ \\
\hline
\end{tabular}

* Beginn sobald Patientin sich vorstellt; \# je nach Mobilität für 6 bis 8 Wochen; + niedermolekulare Heparine; heterozygote Faktor V-Leiden Mutation, heterozygote Prothrombingen-Mutation, milder Protein S- und Protein CMangel; \$ idiopathische venöse Thromboembolien bei Angehörigen ersten Grades; I therapeutische Dosis bei dauerhafter Antikoagulation; \& homozygote Faktor V-Leiden oder Prothrombingenmutation, kombinierte Defekte, ausgeprägte Protein S-, oder Protein C- Mangel, allenfalls Dysfibrinogenämie, Patientinnen mit Antithrombin-Mangel benötigen spezielle Empfehlungen, alllenfalls eine Substitution von Antithrombin ${ }^{22}$; ** Bei Vorhandensein von mindestens drei der folgenden Punkte: Positive Familienanamnese, Alter > 35 Jahre, Rauchen, ausgeprägte Varikosis, Prä-Eklampsie, Dehydratation/ Hyperemesis, Mehrlingsschwangerschaften, lange Geburt, postpartale Blutung, Infektion; doppelt zählen Immobilisation, Adipositas (BMI > 35 kg/m2), Sectio caesare, Begleiterkrankungen, chirurgische Eingriffe in den vergangenen 6 Wochen. 
Tabelle 4: Antikoagulation in der Schwangerschaft - Empfehlungen für Art und Dosis (adaptiert nach ${ }^{4-6,8,11,23}$ )

\begin{tabular}{|c|c|c|}
\hline Schema & Niedermolekulare Heparine & Unfraktioniertes Heparin ${ }^{\#}$ \\
\hline Prophylaktische & 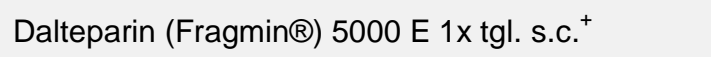 & 5‘000-10‘000 E 12-stündlich s.c. \\
\hline Antikoagulation & $\begin{array}{l}\left.\text { Enoxaparin (Clexane }{ }^{\circledR}\right) 40 \mathrm{mg} 1 \times \mathrm{tgl} \text {. s.c. } \\
\left.\text { Tinzaparin (Innohep }{ }^{\circledR}\right) 4500 \mathrm{E} 1 x \text { tgl. s.c. }\end{array}$ & $\begin{array}{l}\text { 1. Trimenon: 5‘000-7‘500 E s.c. 12-stündlich s.c. } \\
\text { 2. Trimenon: 7’500-10’000 E s.c. } 12 \text {-stündlich s.c. } \\
\text { 3. Trimenon: } 10 \text { '000 E s.c. } 12 \text {-stündlich s.c., es sei } \\
\text { denn die aPTT ist verlängert }\end{array}$ \\
\hline Intermediäre & 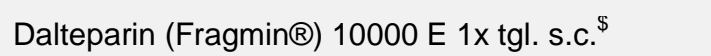 & \\
\hline Dosis & Enoxaparin (Clexane®) $80 \mathrm{mg} 1 \times$ tgl. s.c. ${ }^{\$}$ & \\
\hline \multirow{3}{*}{$\begin{array}{l}\text { Therapeutische } \\
\text { Antikoagulation }\end{array}$} & Dalteparin $($ Fragmin $®) 200 \mathrm{E} / \mathrm{kg} 1 \times \mathrm{tgl}$. s.c. ${ }^{\$ \#}$ & $10 ‘ 000$ E oder mehr 12-stündlich s.c. mit dem Ziel \\
\hline & $\begin{array}{l}\text { Enoxaparin }\left(\text { Clexane }{ }^{\circledR}\right) 1 \text { mg/kg 2x tgl. s.c. } \\
\left.\text { Tinzaparin (Innohep }{ }^{\circledR}\right) 100 \text { E/kg 2x tgl. s.c. }\end{array}$ & $\begin{array}{l}\text { einer aPTT im therapeutischen Bereich ( } 1.5 \text { bis } \\
2.5 \times \text { Ausgangswert) oder anti-Xa } 0.3 \text { bis } 0.6 \mathrm{E} / \mathrm{ml} \\
6 \text { Stunden nach Applikation }\end{array}$ \\
\hline & $\begin{array}{l}\text { Empfehlung: etwa monatliche Bestimmung des } \\
\text { anti Xa-Spiegels (Ziel im peak } 4 \mathrm{~h} \text { nach } \\
\text { zweimalige Applikation per } 24 \mathrm{~h}: 0.6-1.0 \mathrm{E} / \mathrm{ml} \text {; } \\
\text { nach einmalige Application per } 24 \mathrm{~h}: 1.0-2.0 \\
\text { E/ml) }\end{array}$ & \\
\hline
\end{tabular}

\# Alternative bei niereninsuffizienten Patienten oder in anderen Spezialsituationen; ${ }^{+}$bei Patienten > 100kg: 7500 E $1 x$ tgl. s.c.; ${ }^{\circ}$ bei Patienten > 100kg: 60mg 1x tgl. s.c.; ${ }^{\$}$ alternativ

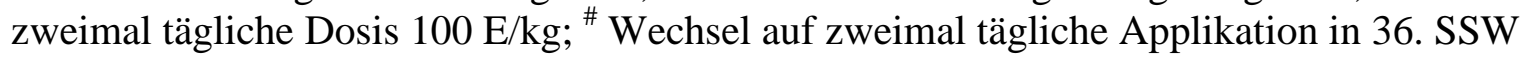
empfohlen; * etwa monatliche Bestimmung des anti Xa-Spiegels empfohlen (Ziel im peak $4 \mathrm{~h}$ nach zweimalige Applikation per 24 h: 0.6-1.0 E/ml; nach einmalige Application per 24 h: 1.0$2.0 \mathrm{E} / \mathrm{ml})$ 
Tabelle 5: Empfehlungen im Hinblick auf östrogenhaltige Kontrazeptiva

Klinische Situation

Stattgehabte venöse Thromboembolien

Asymptomatische Patientinnen

Stark positive Familienanamnese $\left(\geq 2\right.$ Verwandte $1^{\circ}$ mit idiopathischem Ereignis)

Mässige familiäre Belastung (keine Verwandte $1^{\circ}$ ) oder 1 Verwandte $1^{\circ}$ und Thrombophilie

Thrombophilie und unauffällige Familienanamnese

Familienanamnese mit Hochrisiko-Thrombophilie

Klinische Risikofaktoren

(siehe Tabelle 15)
Empfehlung

Meiden*

Meiden*

Individualentscheid+

Individualentscheid*

Meiden*

Individualentscheid\#

* In die Entscheidung einfliessen sollte die Art der Kontrazeptiva. Kombinationspräparate der dritten Generation sind mit einem höheren Risiko verbunden als Medikamente der zweiten Generation; + Bestimmung der Thrombophilieparameter kann jedoch weder die Neigung bestätigen noch sicher ausschliessen; \# Meiden bei Zusammentreffen mehrerer Faktoren empfohlen. 